Sonja Georgi, Julia Ilgner, Isabell lammel, Cathleen Sarti, Christine Waldschmidt (Hg.)

Geschichts-

transformationen

Medien, Verfahren und Funktionalisierungen historischer Rezeption

[transcript] 
Sonja Georgi, Julia Ilgner, Isabell Lammel,

Cathleen Sarti, Christine Waldschmidt (Hg.)

In Zusammenarbeit mit Matthias Emrich,

Dorothea Flothow, Svenja Frank, Marcel Hartwig,

Jacqueline Hylkema, Uta Miersch und Dominik Schuh.

Geschichtstransformationen

Mainzer Historische Kulturwissenschaften | Band 24

E-Book von Julia Ilgner, julia.ilgner@gmx.de

Copyright 2014, transcript Verlag, Bielefeld 
Sonja Georgi, Julia Ilgner, Isabell Lammel,

Cathleen Sarti, Christine Waldschmidt (Hg.)

\section{Geschichtstransformationen}

Medien, Verfahren und Funktionalisierungen

historischer Rezeption

\section{[transcript]}




\section{Bibliografische Information der Deutschen Nationalbibliothek}

Die Deutsche Nationalbibliothek verzeichnet diese Publikation in der Deutschen Nationalbibliografie; detaillierte bibliografische Daten sind im Internet über http://dnb.d-nb.de abrufbar.

\section{() 2015 transcript Verlag, Bielefeld}

Die Verwertung der Texte und Bilder ist ohne Zustimmung des Verlages urheberrechtswidrig und strafbar. Das gilt auch für Vervielfältigungen, Übersetzungen, Mikroverfilmungen und für die Verarbeitung mit elektronischen Systemen.

Umschlagkonzept: Kordula Röckenhaus, Bielefeld

Korrektorat \& Satz: Cathleen Sarti

Printed in Germany

Print-ISBN 978-3-8376-28I5-9

PDF-ISBN 978-3-8394-28I5-3

Gedruckt auf alterungsbeständigem Papier mit chlorfrei gebleichtem Zellstoff. Besuchen Sie uns im Internet: $h t t p: / / w w w . t r a n s c r i p t-v e r l a g . d e$

Bitte fordern Sie unser Gesamtverzeichnis und andere Broschüren an unter: info@transcript-verlag.de 


\title{
Strategien der Geschichtstransformationen in Themenparks
}

\section{FILIPPO CARLÀ \& FLORIAN FREITAG}

\begin{abstract}
Ausgehend von einer Definition von Themenparks als Heterotopien entwickelt der Beitrag ein vierstufiges Modell geschichtstransformatorischer Strategien, die in Themenparks einen affektiven Zugang zu einer ideologisierten, kommodifizierten und präsentifizierten Vergangenheit ermöglichen. Diese grundlegende Neukonzeption von Vergangenheit, die sich allenfalls punktuell an Maßstäben von Authentizität orientiert, gleichwohl jedoch als ungemein wirkmächtig erachtet werden muss, verortet der Beitrag in einer breiteren ästhetischen und kulturellen Entwicklung der Postmoderne, die als ,affective turn " bezeichnet worden ist. Das Modell der geschichtstransformatorischen Strategien wird in der Folge anhand von zwei Fallbeispielen - Main Street, U.S.A. in Disneyland und Grecia in Terra Mítica - illustriert, wobei besonders auf den historischen und kulturellen Kontext der Parks eingegangen wird, der bei Geschichtstransformationen in Themenparks eine kaum zu überschätzende Rolle spielt.
\end{abstract}

\section{Einleitung}

„Here You Leave Today and Enter the World of Yesterday, Tomorrow and Fantasy“: Mit diesen Worten auf einer Tafel am Eingang begrüßt Disneyland in Anaheim, Kalifornien, seit mehr als 50 Jahren seine Besucher. Was damit verdeutlicht wird, ist die Existenz einer Welt jenseits des Eingangs, die der normalen Zeitlichkeit enthoben ist. Ein zentraler Bestandteil dieser anderen Welt ist - wie auch das Motto nahelegt - die Darstellung vergangener Epochen. Diese Darstellungen orientieren sich jedoch nur punktuell an Maßstäben 
von Authentizität; Ziel ist vielmehr die Erschaffung einer Gelegenheit, Geschichte direkt $\mathrm{zu}$ begegnen und $\mathrm{zu}$ erleben. Wie wir im Folgenden zeigen werden, kommt es in diesem Prozess zu klaren Formen der, Geschichtstransformation'.

In Transformation. Ein Konzept zur Erforschung kulturellen Wandels (2011) definieren Lutz Bergemann, Martin Dönike, Albert Schirrmeister, Georg Toepfer, Marco Walter und Julia Weitbrecht ,Transformationen“ als

komplexe Wandlungsprozesse [...], die sich zwischen einem Referenz- und einem Aufnahmebereich vollziehen. Aus dem Referenzbereich wird durch einen (nicht notwendig personal zu verstehenden) Agenten ein Aspekt ausgewählt, wobei im Akt der Aneignung nicht nur die Aufnahmekultur modifiziert, sondern insbesondere auch die Referenzkultur konstruiert wird. Diese enge Beziehung von Modifikation und Konstruktion ist wesentliches Merkmal transformatorischer Prozesse, die sowohl diachron als auch synchron verlaufen können. ${ }^{1}$

Laut Laura Bieger ist Transformation darüber hinaus eine der zwei Komponenten, aus denen die Bildpolitik thematisierter, immersiver Räume (zu denen auch Themenparks zählen) besteht:

(1) dem Transfer von kulturellem Material und

(2) dessen Transformation gemäß den Anforderungen und Bedürfnissen des neuen Kontextes, in dem dieses Material wirksam werden soll; zwei Aspekte, die sich in ihrer Produktivität nicht voneinander trennen lassen, sondern unauflöslich ineinander greifen und sich gegenseitig hervorbringen. ${ }^{2}$

Mit Bergemann u. a. und Bieger lassen sich Geschichtstransformationen (speziell in Themenparks, aber auch generell) somit als zweite Komponente der Bildpolitik immersiver Themenräume begreifen, die wiederum als Produkt zweier Mechanismen gefasst werden kann: (1) einer Modifikation spezifischer historischer Ereignisse oder narrativer Überlieferungen und (2) einer generellen Neukonzeptionalisierung von Vergangenheit.

Letzterer Bestandteil entspricht einer verbreiteten ästhetischen und kulturellen Entwicklung der Postmoderne, die sich auch in neueren Konzeptionen

1 Bergemann u. a., 2011, S. 39. In diesem Sinne kann man Rezeptionsprozesse auch als „transkulturelle Dynamiken“ (CARLÀ, 2014, S. 33-38) oder als „Übersetzung" (BIEGER, 2007, S. 18) bezeichnen.

2 BIEGER, 2007, S. 52. 
von Museen findet und die von Vanessa Agnew als „affective turn“ identifiziert worden ist. ${ }^{3}$ Laut Agnew bezeichnet dieser ,the collapsing of temporalities and an emphasis on affect, individual experience and daily life rather than histor-ical events, structures and processes ${ }^{\text {}}$. Genau deshalb sind die Begriffe der Authentizität und der historischen Genauigkeit bei solchen Rezeptionsformen auch irrelevant - diese Aneignungen sind immer ,echt ${ }^{6}$, aber ,their realism is not that of a lost, real past but of real sensual impressions and emotions in the present, which engage visitors and engender meaningful feelings" $"$.

Der affective turn ist generell in den postmodernen Prozessen eines Verlusts der linearen Zeitwahrnehmung (und der Wahrnehmung der Gegenwart als vorläufigen Punkt zwischen Vergangenheit und Zukunft) und einer ,Sehnsucht' nach einer kompletten ,Präsentifizierung ' zu verorten; Phänomene, die u. a. von Frederic Jameson, Lucian Hölscher und Hans Ulrich Gumbrecht beschrieben worden sind. ${ }^{6}$ Dieser Verlust der Linearität der Zeit und das Interesse für eine präsentifizierte Vergangenheit sind keinesfalls gleichbedeutend mit einem sinkenden Interesse an der Vergangenheit per se; ganz im Gegenteil scheint die ,Leidenschaft" für vergangene Epochen heutzutage verbreiteter zu sein als je zuvor. ${ }^{7}$ In diesem Sinne sind Themenparks von der Vergangenheit inspiriert; ihr Besuch stellt jedoch nicht, wie es Disneys Motto nahelegt, eine Reise in die Vergangenheit dar, sondern eine echte Vergegenwärtigung, einen Teil der gegenwärtigen Erfahrung.

Es ist deshalb wichtig, die Relevanz der Themenparks in der Konstruktion der Vergangenheit sowie im Verständnis des postmodernen Ansatzes zur Geschichte nicht zu unterschätzen - auch aufgrund des postmodernen Verlusts einer klaren Differenzierung zwischen ,Hoch'- und ,Populärkultur'. Die spezifischen Formen, die die Darstellung der Geschichte hier einnimmt, erzielen eine immense Wirkung und beeinflussen nachhaltig das Geschichtsbild eines

3 Vgl. AgNEw, 2007. Vgl. auch Raphael Samuels Definition von „Retrochic“, der ihm zufolge die Unterscheidung zwischen Original und Kopie verwischt (SAMUEL, 2012, S. 112).

4 AGNEw, 2007, S. 299.

5 Für eine Definition des Authentizitätsbegriffs in Bezug auf die ,Pastness', vgl. jetzt HOLTORF, 2013. Vgl. auch DERS., 2005, S. 135f.; DERS., 2010, S. 28f. Zur ,Unerheblichkeit' des traditionellen Authentizitätsbegriffes in Studien der popular history, vgl. auch DE GROOT, 2009, S. 110-112.

6 Vgl. u. a. JAMESON, 1996, S. 16-25; HÖLSCHER, 2002, S. 140-142; GuMBRECHT, 2004, S. 118-125. Vgl. auch SAmuel, 2012, S. 190, der die Geburt der living history in die späten 1960er Jahre datiert.

7 Vgl. SAmuel, 2012, S. 25. 
breiten Publikums. ${ }^{8}$ Sie spielen so, laut Jerome de Groot, eine zentrale Rolle in den Prozessen der „Kommodifizierung der Geschichte“9 immer betrachten sollten, im Bewusstsein, dass die akademische Perspektive nur eine partielle ist und dass Formen der leisure history einen wichtigeren Beitrag zur Konstruktion der verbreiteten Bilder der Vergangenheit leisten. ${ }^{10}$ Man sollte in diesem Zusammenhang nicht vergessen, dass die Besucherzahlen der Themenparks (allein Disneyland hat etwa 14 Millionen Gäste pro Jahr) ihrem Geschichtsbild eine Popularität geben, ,with which professional historians are unable to compete in terms of either the mode of presentation or the numbers of people touched by it." 11

Ziel dieses Aufsatzes ist es zunächst, Themenparks zu definieren, um sodann die Strategien, die den im Themenpark stattfindenden disjunktiven geschichtstransformatorischen Prozessen zugrunde liegen, ${ }^{12}$ genauer darzustellen und ihre soziale und politische Relevanz zu erörtern. Im Anschluss werden wir diese Strategien anhand zweier konkreter Fallbeispiele, Disneyland, Kalifornien sowie Terra Mítica in Spanien, zu verschiedenen historischen Epochen näher vorstellen, um einen Einblick in die zur Verfügung stehenden methodologischen Instrumente einer solchen Untersuchung zu geben. Im Zentrum der Untersuchung stehen somit nicht einzelne historische Epochen selbst, sondern vielmehr die zwei Seiten der Allelopoiese oder der wechselseitigen Produktion historischer Epochen in Themenparks: die Konstitution der Gegenwart durch die Vergangenheit und die Konstruktion der Vergangenheit durch die Gegen-

8 Vgl. SAmuel, 2012, S. 160: „The new version of the national past [...] is inconceivably more democratic than earlier ones, offering more points of access to , ordinary people.““

9 Vgl. De Groot, 2009, S. 4f.

10 Vgl. auch Jordanova, 2006, S. 33f. Eine konservativere Sicht der Trennung zwischen ,professioneller' und ,populärer' Geschichte ist auch heute noch z. T. vertreten. So bezeichnete etwa Samuel noch 1996 die living history als ,offensive for the professional historian“" (SAMUEL, 2012, S. 197), auch wenn er ein Interesse von professionellen Historikern für verschiedene Geschichtsformen als notwendig ansieht (vgl. EBD., S. 15).

11 BRYMAN, 1995, S. 142. Für Daten bezüglich der Verkaufszahlen populärwissenschaftlicher historischer Publikationen in England, vgl. z. B. DE Groot, 2009, S. 31f.

12 Bergemann u. a., 2011, S. 49 verwenden den Begriff ,Disjunktion“ zur Bezeichnung einer ,Transformation, bei der ein Inhalt der Referenzkultur in die Form der Aufnahmekultur gekleidet wird oder ein Inhalt der Aufnahmekultur eine in der Referenzkultur verwendete Form erhält". 
wart, ${ }^{13}$ d. h. die Funktionen historischer Epochen ,in den kulturellen und künstlerischen, politischen und wissenschaftlichen Selbstpositionierungen von Nachfolgekulturen"14 sowie die Mittel und Strategien, die insbesondere in Themenparks verwendet werden, um historische Epochen für solche Selbstpositionierungen fruchtbar zu machen.

\section{Themenparks: Eine Definition}

Themenparks sind ,abgeschlossene, großflächig angelegte, künstlich geschaffene, stationäre Ansammlung[en] verschiedenster Attraktionen, Unterhaltungsund Spielangebote, die sich fast immer außerhalb großer Städte/Metropolen befinde[n], die ganzjährig geöffnet und kommerziell strukturiert [sind]“. ${ }^{15}$ Themenparks zählen zu den Erlebniswelten ${ }^{16}$ und insbesondere zur Unterkategorie der themed environments, deren konstitutives Merkmal ,die thematische Geschlossenheit [darstellt], d. h., dass entweder der ganze Vergnügungspark oder aber einzelne, in sich geschlossene Teile auf bestimmte Motive, Themata, Figuren usf. sowie deren Wiedererkennbarkeit angelegt sind". ${ }^{17}$

Aus architektonischer und stadtplanerischer Sicht wurden Themenparks darüber hinaus häufig als ,Utopien' bezeichnet. ${ }^{18}$ Auch Historiker wie Michael Wallace verwendeten aufgrund ihrer bereinigten Versionen von Vergangenheiten diese Beschreibung. ${ }^{19}$ Themenparks zeichnen sich jedoch gerade nicht durch alternative Projekte zur Konstruktion einer verbesserten Gesellschaft aus, die für die Utopie konstitutiv sein sollten. Eine Definition als Utopie ist deshalb nur in einem sehr breiten Sinne annehmbar; zutreffender scheint es dagegen, Michel Foucaults Begriff der Heterotopie bzw. der Heterochronie heranzuziehen. ${ }^{20}$ Foucault beschrieb in der Tat Heterotopien in Kontrast zu Utopien als eine bestimmte Art von lokalisierbaren Orten, die von der übrigen

13 Vgl. EBD., S. 43.

14 EBD., S. 41.

15 Kagelmann, 1993, S. 407.

16 Vgl. SteineCKe, 2000.

17 Kagelmann, 1993, S. 408.

18 Vgl. z. B. KING, 1981.

19 Vgl. Wallace, 1985.

20 Vgl. PhiLiPs, 1999, S. 91. 
Welt strikt abgetrennt sind, trotzdem jedoch Bezug auf die Welt nehmen alles Eigenschaften, die auf Themenparks zutreffen. ${ }^{21}$

Auch die von Foucault zur Beschreibung von Heterotopien entwickelten Prinzipien der Heterotopologie lassen sich größtenteils auf Themenparks anwenden. ${ }^{22}$ Insbesondere sind folgende Prinzipien zutreffend: die Möglichkeit der Vereinigung mehrerer normalerweise zeitlich und räumlich getrennter Kontexte (etwa durch die Präsenz mehrerer Themenbereiche in einem Park); eine strikte Abtrennung von der übrigen Welt und Zeit(lichkeit); der beschränkte Zutritt, der durch den Kauf einer Eintrittskarte ritualdynamisch markiert wird. Schließlich haben laut Foucault Heterotopien eine illusorische oder eine kompensatorische Funktion, d. h. entweder entlarven sie die reale Welt als Illusion oder entwerfen eine ideale Gegenwelt zur realen Welt - beide Funktionen sind Themenparks zugeschrieben worden. ${ }^{23}$

\section{Strategien der Geschichtstransformationen in Themenparks}

Geschichtstransformationen in Themenparks beruhen auf vier Modifikationsstrategien:

1. Selektion. Zunächst werden die im Themenpark darzustellenden Epochen und die innerhalb dieser Epochen darzustellenden Elemente ausgewählt. Sie sind im Normalfall (auch durch andere Formen der modernen Rezeption) schon bekannt und deshalb einfach rezipierbar. Häufig werden auch Epochen und Themen ausgewählt, die implizite oder explizite Anknüpfungspunkte zu gegenwärtigen Identitätsfragen bieten und eine besondere Rolle im kulturellen Gedächtnis der jeweiligen Zielgruppe des Parks spielen. Außer in Fällen von dark theming ${ }^{24}$ werden politisch und sozial sensitive Themen wiederum mit Blick auf das Zielpublikum ausgeblendet. ${ }^{25}$ Der Themenpark greift so vorhandene Stereotype auf und perpetuiert diese weiter. ${ }^{26}$

21 Vgl. Foucault, 2005, S. 1574f.

22 Vgl. EBD., S. 1575-1580.

23 Zur illusorischen Funktion vgl. BAUdRILlard, 1981, S. 12f. Der Fokus auf die kompensatorische Funktion hat zur bereits erwähnten Klassifizierung von Themenparks als Utopien geführt.

24 Vgl. LuKAS, 2007, S. 276-280.

25 Bergemann u. a., 2011, S. 51 bezeichnen diese Form der Transformation als „Ignoranz“, eine „Transformation, die Tatsachen oder Sachverhalte nicht beachtet. 
Ein Beispiel bietet die Darstellung Griechenlands im Europa-Park in Rust. Griechenland wird hier, anders als viele andere Länder, nicht als moderner europäischer Staat dargestellt, sondern als antiker Kulturraum. So wird einerseits das Bild Griechenlands als Ursprung der europäischen Kultur betont und damit wird die Rolle des Landes im kulturellen Gedächtnis der ganzen EU aufgewertet; andererseits werden dadurch aber auch politisch problematische Themen im Zusammenhang mit der modernen Geschichte Griechenlands (Unabhängigkeitskriege, Konflikte mit der Türkei, militärische Diktatur) ausgeblendet. Die Entscheidung des Europa-Parks, Griechenland durch seine antike Kultur darzustellen, fußt auch auf der großen Bekanntheit dieser Kultur und ihrer Formen (Architektur, Epen, Mythen) und auf der bereits existierenden medialen Rezeption - etwa in Filmen oder Gemälden. Auch innerhalb des gewählten Zeitraums kam es zu einer Selektion der Materialien, die nach Rust „transferiert“ wurden: Ausgeblendet wurden Themen wie z. B. die Sklaverei, die allesamt als nicht, familienfreundlich' gelten würden.

2. Abstraktion. Der Themenpark übersetzt diese Stereotypen in multimediale thematische Darbietungen, die in ihrer Typik möglichst viele Menschen ansprechen. In der Themenparkarchitektur z. B. werden durch das Aufgreifen bzw. die Amalgamierung historischer Originale visuelle kleinste gemeinsame Nenner bzw. echte architektonische ,Piktogramme ' geschaffen, die evokativer sein sollen als einfache Kopien bekannter Gebäude. Dies stellt nicht notwendigerweise eine neue Entwicklung dar: Gerade im Hinblick auf visuelle Rezeption stellt etwa Samuel fest, dass ,historical illustration is [...] one of the most conservative of art forms. The same stock figures seem to appear in an astonishing variety of contexts“. 27

Um bei dem Beispiel des griechischen Themenbereichs in Rust zu bleiben: Die Station der dortigen Wasserachterbahn Poseidon stellt einen altgriechischen Tempel dar, der von den Fronton-Skulpturen zu den dorischen Kapitellen alle Bestandteile enthält, die gemeinhin einem griechischen Tempel zugeschrieben werden (ohne einen konkreten altgriechischen Tempel zu reproduzieren), auch wenn dieses verbreitete Bild ein modernes Produkt ist, das im Widerspruch zu archäologischen Rekonstruktionen steht. Es ist insbesondere

Dies kann entweder den bewussten Verzicht auf eine Auseinandersetzung oder auch die (unbewusste) Unfähigkeit meinen, etwas zur Kenntnis zu nehmen“.

26 Vgl. PHILIPS, 1999, S. 106.

27 SAMUEL, 2012, S. 32. 
bekannt, dass griechische Tempel bunt bemalt waren; in dieser Form wären sie aber für ein breites Publikum nicht wiedererkennbar. ${ }^{28}$

Durch die Kombination von Selektion und Abstraktion kommt es Bieger zufolge zu einer „Fiktionalisierung“, die, wie erwähnt, z. T. fälschlicherweise als ,Utopisierung“ empfunden worden ist: „Störendes wird ausgeblendet oder verklärt, Vorhandenes wird nach bestimmten Vorgaben inszeniert, affirmativ mit Bedeutung und Vorstellungen aufgeladen und in einem neuen, komprimierten Bezug räumlich verdichtet. “29

3. Immersion. Die Vergegenwärtigung verschiedener Zeitebenen wird dadurch erreicht, dass diese nicht wie etwa im Museum in einer fragmentarischen Form dargeboten, sondern dass alle Sinne gleichzeitig angesprochen werden und Besucher damit z. B. gezielt durch Musik, Architektur, Geruch, Geschmack in eine andere Zeit versetzt werden. Geschichte wird dadurch , affektiv ' am eigenen Körper erfahrbar und erlebbar gemacht. ${ }^{30}$ Eine weitere wichtige Voraussetzung für die Immersion der Besucher stellt die strikte visuelle Abgrenzung der Themenwelt von anderen Themenbereichen des Parks und von der Außenwelt dar.

Themenparks sind selbstverständlich nicht die einzigen immersiven Medien; in ihnen fällt jedoch der Grad an Immersivität besonders hoch aus, ${ }^{31} \mathrm{da}$ etwa im Gegensatz zum Film oder zum Videospiel Rezeptionsort und thematischer Raum - und damit auch Rezeptionszeit und dargestellte Zeit - zusammenfallen. Die Vergangenheit im Themenpark ist eine vergegenwärtigte Vergangenheit.

4. Transmedialisierung. Zur Immersion der Besucher setzt der Themenpark andere immersive Medien wie z. B. Theater oder Film ein. Auch weniger immersive Medien wie etwa Skulptur oder Malerei werden im Kontext des Themenparks zu Teilen der immersiven Umwelt. Die einzelnen Medien werden im Themenpark zu einem sog. Intermedia Text verflochten. In einer Reihe von Publikationen von 1992 bis 2007 unterscheidet Claus Clüver zwischen Multimedia, Mixed-Media und Intermedia Texten. Themenparks sind in diesem Modell zu der letzten Kategorie zu zählen, da ihre einzelnen Elemente nicht voneinander getrennt werden können, ohne ihre Kohärenz und Selbstgenügsamkeit zu verlieren. ${ }^{32}$

28 Vgl. Holtorf, 2013, S. 433f.

29 BIEGER, 2007, S. 153.

30 Vgl. SAMuel, 2012, S. $175 f$.

31 Vgl. EBD., S. 177f.

32 Vgl. ClüVER, 2007. 
Durch diese Mechanismen der Modifikation werden den Besuchern bestimmte Botschaften, häufig mit einem starken politischen bzw. ideologischen Inhalt, vermittelt; das Produkt ist eine völlige Neukonzeptionalisierung von Vergangenheit. Da die Inhalte dieser Botschaften dem Publikum jedoch nicht explizit und argumentativ, sondern in einer direkt erlebbaren, affektiven Form vorgestellt werden, werden sie , verschleiert', naturalisiert und objektiviert. Es ist ein typisches Merkmal der ,populären' und ,kommodifizierten' Geschichte, teleologische, erklärende, positivistische Ansätze zu bevorzugen und sich dadurch u. a. mit Fragen der nationalen und ethnischen Identität zu befassen, die von akademischen Historikern als , problematisch ' empfunden werden. ${ }^{33}$ Anders als häufig behauptet, ${ }^{34}$ schließen sich somit affektive Ansätze und die Existenz von zugrunde liegenden master narratives in Geschichtsdarstellungen - und damit auch master narratives und Postmodernität - nicht grundsätzlich aus.

Ein Beispiel, das all dies verdeutlichen kann, sind die Parks, die die nationale Identität bestimmter Länder, die sehr starke regionale Identitäten aufweisen, untermauern, indem sie Merkmale, Denkmäler und sogar Traditionen der verschiedenen Regionen zusammenfügen, um den Besuchern eine explizite Botschaft der ,Einheit in der Vielfältigkeit' zu vermitteln. Was in Themenparks konstruiert wird, ist die Idee einer gemeinsamen Geschichte, die sich durch die Summe der lokalen Geschichten und im Respekt für die lokalen Traditionen aufbaut. $\mathrm{Zu}$ dieser Kategorie gehören u. a. sowohl Taman Mini Indonesia Indah, ein Park, den Präsident Sukarto persönlich förderte und der 1975 in Jakarta eröffnet wurde, ${ }^{35}$ sowie Italia in Miniatura, ein Park, der 1970, im Zuge einer Debatte über das angeblich mangelhafte nationale Identitätsgefühl der Italiener, in der Nähe von Rimini eröffnet wurde.

\section{Fallbeispiel 1: Main Street, U.S.A. (Disneyland, Kalifornien)}

Bei unserem ersten Fallbeispiel handelt es sich um einen Themenbereich von Disneyland in Kalifornien, ein Park, der nicht nur ähnlich wie Taman Mini Indonesia Indah und Italia in Miniatura mit regionalen Identitäten einer Nation spielt, sondern mit seiner über 55jährigen Geschichte und seinen bis zu

33 Vgl. De Groot, 2009, S. 21.

34 Vgl. z. B. SAMUEL, 2012, S. 195f.

35 Vgl. SCHLEHE/UiKe-BormanN, 2010, S. 73-82. 
14 Millionen Besuchern jährlich zu den ältesten und erfolgreichsten Themenparks überhaupt zählt. Der Park ist nach dem Magic Wand-Prinzip aufgebaut, ${ }^{36}$ d. h. der Haupteingang ist durch einen Korridor mit dem Zentrum des Parks verbunden, von dem aus sich verschiedene Themenbereiche gleich Tortenstücken abspreizen. Bei dem Korridor handelt es sich im Fall von Disneyland um die sog. Main Street; das Zentrum des Parks wird markiert durch das Sleeping Beauty Castle, das auch als Wahrzeichen des Parks fungiert; bei den einzelnen Themenbereichen handelt es sich um Adventureland, Frontierland, Fantasyland und Tomorrowland.

Aus operationeller Sicht nimmt Main Street innerhalb des Parks mehrere Funktionen ein: Sie kanalisiert den Besucherstrom vom Eingang in das Zentrum des Parks und stellt damit den einzigen Themenbereich dar, den alle Besucher zumindest beim Betreten und Verlassen des Parks betreten müssen. In Main Street finden sich zudem wesentliche Besucherservices wie das Fundbüro oder die Erste-Hilfe-Station des Parks und sie bietet den Besuchern auch die größte Anzahl an Shops und Restaurants. Attraktionen und Fahrgeschäfte sind dagegen nur spärlich vorhanden und beschränken sich vor allem auf Paraden und Street Entertainment.

Thematisch rekurriert Main Street auf die amerikanische Kleinstadt des Mittelwestens um die Jahrhundertwende. Zeitlich lässt sich Main Street genauer zwischen 1896 und 1908 verorten, denn die zahlreichen Flaggen, die die Gebäude schmücken, zeigen 45 Sterne, ebenso wie die amerikanische Flagge vom 4. Juli 1896 (dem Jahr des Beitritts Utahs als 45. Staat zu den Vereinigten Staaten) bis zum Wechsel der Anzahl der Sterne am 3. Juli 1908 anlässlich des Beitritts von Oklahoma als 46. Staat. Geografisch wird Main Street in Disneyland-Metatexten in der Region des Mittelwestens verortet, indem betont wird, der Themenbereich sei von der Kleinstadt Marceline, Missouri, inspiriert, in der Walt Disney einen Teil seiner Kindheit verbrachte. ${ }^{37}$ Im Park selbst jedoch finden sich keinerlei Hinweise, die eine genaue geografische Verortung in den Vereinigten Staaten zulassen würden. Vielmehr verweist der Namenszusatz „U.S.A.“ darauf, dass es sich um eine typisch amerikanische Kleinstadt handeln soll, unabhängig von regionalen Differenzen.

Main Street fungiert in der Tat sowohl operationell wie auch thematisch als ein sozialer Schmelztiegel, der die Besucher unabhängig von ihrer Herkunft, Ethnie oder Klasse vor allem als (weiße, protestantische, bürgerliche) US-

36 Vgl. Mitrasinovic, 2006, S. 127.

37 Vgl. z. B. SHANNON, o. J., S. 42. 
Amerikaner anspricht. Dies wird in etwas anderer Akzentuierung auch in der Pariser Version von Main Street deutlich, wo den Besuchern nicht mehr ihre jeweils unterschiedlichen Heimatländer, sondern Disneyland identifikatorisch als gemeinsamer Bezugspunkt dienen soll. ${ }^{38}$ Einerseits werden durch das notwendige Durchschreiten der Main Street Unterschiede zwischen den Besuchern rituell nivelliert. Andererseits rekurriert Main Street mit ihrem Fortschrittsnarrativ auf die klassische Selbstdefinition der USA als Land der unbegrenzten Möglichkeiten: In thematischen Details wie dem Nebeneinander von Gaslaternen und elektrischen Leuchten sowie von pferdegezogenen Wagen und Automobilen oder Hinweisen auf die Suffragetten-Bewegung erzählt Main Street eine Geschichte des technologischen und sozialen Fortschritts. Die Jahrhundertwende in den USA wird so als eine Zeit der wirtschaftlichen Prosperität und deshalb des unbegrenzten Optimismus dargestellt und damit zu einem paradigmatischen Beispiel der amerikanischen Zivilreligion gemacht. Wie Walt Disney selbst es formuliert hat: „[F]or those of us who remember the carefree time it recreates, Main Street will bring back happy memories. For younger visitors, it is an adventure in turning back the calendar to the days of grandfather's youth. “39

Dafür jedoch werden nach den schon erwähnten Mechanismen der Strategie der Selektion zahlreiche Auslassungen in Kauf genommen. So schreibt etwa der Historiker Mike Wallace in Bezug auf Main Street:

The decades before and after the turn of the century had their decidedly prosperous moments. But they also included depressions, strikes on the railroads, warfare in the minefields, squalor in the immigrant communities, lynching, imperial wars, and the emergence of mass protests by populists and socialists. This history has been whited out, presumably because it would have distressed and repelled visitors. ${ }^{40}$

Außer den von Wallace genannten Aspekten wurden außerdem noch das problematische Verhältnis der Kleinstadt zum landwirtschaftlichen Hinterland

38 Vgl. die Beschreibung der Pariser Main Street, U.S.A. in einem Souvenirbuch von 1994: „You have come from all over the world. Whether you have left behind the hum and drone of bus and train, or parked your car under the watchful eyes of Alice, Bambi or Donald in Guest Parking, the moment you reach Euro Disneyland Resort you are in a different world." (SHRAGER, 1994, S. 5).

39 Zit. n. SHAFFer, 2010, S. 126.

40 Wallace, 1985, S. 137. 
oder die repressiven, ultrakonservativen Sozialstrukturen der amerikanischen small town, wie sie etwa in der sog. Revolt of the village-Literatur der 1920er und 1930er Jahre zum Ausdruck gebracht wurden, ${ }^{41}$ völlig ausgeblendet.

Die Idealisierung des ,Goldenen Zeitalters' der Jahrhundertwende wird visuell durch die Mechanismen der Abstraktion weiter verdeutlicht. Die hyperviktorianische Architektur der Main Street, in der bestimmte Elemente, wie die ornamentale Dekoration der Häuser, überhöht, bestimmte Gegenstände (etwa Strommasten) entfernt und weitere Bestandteile, die damals nicht vorhanden waren, aber zum zeitgenössischen Bild einer sauberen und geordneten Stadt gehören (z. B. Bäume), hinzugefügt wurden, hat mit dem tatsächlichen, ungeplanten Erscheinungsbild amerikanischer Kleinstädte um 1900 wenig gemein. Stattdessen ergibt sich durch eine Abstimmung der Gebäudedimensionen und der Farbpalette ein äußerst harmonisches Gesamtbild.

Die Kleinstadtatmosphäre in Main Street wird jedoch nicht nur durch Architektur erschaffen, sondern auch durch eine klare Abgrenzung von der AuBenwelt und von den anderen Themenbereichen, die von Main Street aus nicht zu sehen sind. Dieses erste Mittel der Immersion wird darüber hinaus durch gezielt ausgewählte Hintergrundgeräusche, Gerüche und Geschmäcke ergänzt. Neben einem Soundtrack, der u. a. Stücke der von Randy Newman komponierten Filmmusik zu Miloš Formans Film Ragtime (1981) enthält, hören die Besucher etwa verschiedene Stimmen aus den Gebäuden, die offensichtlich den Bewohnern von Main Street zugeordnet werden sollen sowie das Geklapper von Pferdehufen. Die Luft ist erfüllt vom Geruch von Kaffee, Backwaren, Popcorn und Zuckerwatte, den zahlreiche, gleichmäßig über den Themenbereich verteilte Verkaufsstände und Geschäfte ausströmen und der zum Verzehr anregen soll (der Pferdemist hingegen wird schnellstmöglich beseitigt).

In der Gestaltung dieser immersiven Darbietung greift Main Street auf verschiedene Medien zurück, von denen hier exemplarisch der Film herausgegriffen werden soll. Zahlreiche Forscher haben erläutert, wie sich Disneyland insgesamt und Main Street im Besonderen Elementen der filmischen mise-enscène (erzwungene Perspektive in der Architektur der Gebäude), mise-encadre (geschlossene Bildformen durch geschickte Landschaftsgestaltung und Anordnung der Gebäude) und der mise-en-chaîne (die bereits erwähnte Film-

41 Vgl. HILFER, 1969. 
musik sowie das an eine filmische Eröffnungssequenz erinnernde rituelle Durchqueren der Main Street auf das zentrale Schloss hin) bedienen. ${ }^{42}$

Die multisensorische, transmediale und immersive Repräsentation der amerikanischen Kleinstadt der Jahrhundertwende schafft eine radikal neue Vergangenheit, eine Vergangenheit, so Jon Wiener, ,without tenements or poverty or urban class conflict" ${ }^{43}$ die den Trend zur Suburbanisierung amerikanischer Städte und insbesondere Los Angeles' zur Zeit der Entstehung Disneylands in den 1950er Jahren aufgreift und dessen Wurzeln in der amerikanischen Kleinstadt der Jahrhundertwende verortet. ${ }^{44}$ Dass es sich hierbei um eine Disneyspezifische Neukonzeptionalisierung der Vergangenheit handelt, wird im Park durchaus angedeutet: Bei den auf den Fenstern der Main Street aufgemalten Namen der ,Bewohner" der Gebäude handelt es sich allesamt um die Namen von Designern, Komponisten und Künstlern, die an der Entstehung von Disneyland beteiligt waren, darunter auch Walt Disney selbst. Die Vergangenheit der Vereinigten Staaten wird hier gleichsam zur Vergangenheit Disneylands.

\section{Fallbeispiel 2: Grecia (Terra Mítica, Spanien)}

Das zweite Fallbeispiel, das hier untersucht werden soll, stellt der Park Terra Mítica dar, der 2000 in Benidorm, Spanien, eröffnet wurde. Weniger erfolgreich als Disneyland (der Park hatte bereits mehrfach erhebliche finanzielle Probleme, wurde z. T. geschlossen und hält sich nur mit Schwierigkeiten über Wasser: 2012 wurde er für einen äußerst niedrigen Preis verkauft und in zwei verschiedene Parks geteilt) ${ }^{45}$ erreichte er mit seinen Geschichtsbildern in den ersten Jahren nach der Eröffnung dennoch ca. 2 Millionen Menschen pro Jahr - viel mehr als die Leser eines erfolgreichen wissenschaftlichen Buchs. Im Jahr 2010 war die Besucherzahl allerdings auf 520.000 abgesunken. ${ }^{46}$

42 Vgl. Marling, 1991, S. 197; KING, 1981, S. 127; FJellman, 1992, S. 257; LAINSBURY, 2000, S. 83; LuKAS, 2008, S. 157; FINCH, 2011, S. 456; LEe/MADEJ, 2012, S. 98.

43 WIENER, 1994, S. 134.

44 Vgl. Avila, 2004.

45 Diese Schwierigkeiten haben mehrere Änderungen und Umgestaltungen des Parks verursacht; was hier beschrieben wird, bezieht sich auf den Stand des Parks während unserer Feldforschung im November 2012.

46 http://www.parksmania.it/2011/12/28/terra-mitica-la-regione-vende-la-sua-quotadi-proprieta/, 15.8.2013. 
Thema des Parks sind die Kulturen des Mittelmeerraums - insbesondere die Antike. Die fünf als Rundgang ${ }^{47}$ angeordneten Themenbereiche bilden eine grobe chronologische und geografische Annäherung an die spanische Kultur, deren ,Entstehungsgeschichte" damit thematisiert wird. So findet man am Eingang Egipto, danach Grecia, Las Islas, einen zweiten griechischen Bereich, der einen stärkeren Bezug auf das Mittelmeer, Kolonisationsphänomene und Reisen nimmt, Roma und letztlich Ibéria, wo Spanien im Mittelalter und in der frühen Neuzeit dargestellt wird. Das Geschichtsbild, das hier angeboten wird, und seine identitätsstiftende Funktion werden so sehr deutlich: Genau um das Jahr 2000 - zu einem Zeitpunkt, als die Diskussionen über die Existenz und die Bestandteile einer , europäischen Identität ' und über die Rolle der einzelnen Nationalkulturen innerhalb dieser Identität besonders heftig waren ${ }^{48}$ - setzte der Park gegen die Idee einer solchen gemeinsamen Identität die Homogenität bzw. Einheit der Kulturen des Mittelmeers - deren Höhe- und teleologischen Endpunkt Spanien darstellt.

Parallel zum vorherigen Fallbeispiel wird auch hier ein besonderer Bereich näher analysiert, um die Formen und Funktionen der dort operierenden Geschichtstransformationen besser zu definieren. Es handelt sich um den griechischen Bereich, ein zentrales Bindeglied in der Anlage, das nicht vermieden werden kann und auch schon vom Eingang aus aufgrund seiner erhöhten Lage und seines Farbschemas als visueller Magnet wirkt. Der Bereich zeigt eine besonders hohe Attraktionsdichte und hat keine Abzweigungen - lässt deshalb den Besuchern keine Freiheit, ihren Weg frei zu gestalten.

Die Kultur Altgriechenlands wird chronologisch dargestellt: Der Eingang zu dem Bereich stellt die mykenische und die minoische Kultur dar; danach führt der Weg der Besucher bergauf, um eine Art Akropolis zu erreichen, die die Welt der klassischen Polis repräsentiert. Die Strategie der Selektion operiert hier zweifach: Einerseits, indem die architektonischen und dekorativen Formen eine Auswahl berühmter griechischer Gebäude und Statuen (z. B. die Statue der Athena Promachos, das Erechtheion oder den Dornauszieher) darstellen, schon existierende und stereotypisierte Rezeptionsformen bestätigen und damit einen hohen Grad an Wiedererkennbarkeit erreichen. Andererseits dadurch, dass unter den verschiedenen Aspekten der antiken griechischen Kultur ein klarer Fokus auf religiöse und mythologische Aspekte gesetzt wird. Wie im Falle von Main Street werden politische und soziale Themen (hier

47 Dieses Modell wird von Mitrasinovic, 2006, S. 139, als Loop Pattern bezeichnet.

48 Vgl. SHORE, 1996. 
z. B. Demokratie, Sklaverei, Krieg) ausgeklammert; stattdessen dient als Brücke zur heutigen Welt die Repräsentation des antiken Sports mit einem eindeutigen Verweis auf die Olympischen Spiele.

Dieser Verweis materialisiert sich durch die Gruppierung der Attraktion Templo de Kinetos, einer Reproduktion des Tempels des Zeus von Olympia (in der sich auch eine Rekonstruktion der Phidias-Kultstatue befindet), mit einer photo opportunity (einer Kulisse, vor der sich die Besucher fotografieren können), die aus einem Gewinnerpodest und einer Exedra mit Reproduktionen antiker Statuen besteht. Das Podest macht eine antike Idee in einer modernen Form greifbar: Auch wenn solche Podeste in der Antike nie existierten und das Podest mit seinen römischen Ziffern bestenfalls einen antiken, keinesfalls aber griechischen Eindruck erweckt, ${ }^{49}$ verweist es dennoch direkt auf die antike Welt des Sports. Diese Referenz wird durch die Auswahl der Statuen verstärkt, die den Hintergrund des Podests bilden. Jede der neun Statuen stand entweder bereits in der Antike im Zusammenhang mit sportlichen Aktivitäten (Lysippos' Anaxyomenos, Polykletos' Diadumenos, Naukydes' Diskophoros sowie eine Statue, die stark an den Athleten des Stephanos erinnert) oder referiert in der modernen Wahrnehmung darauf, indem sie ein Ideal des männlichen Körpers thematisiert. Wenn für Polykletos' Doryphoros, vielleicht die berühmteste griechische Statue, in der Tat eine Interpretation als athletische Figur von einigen Forschern vorgeschlagen worden ist ${ }^{50}$ gilt dies keinesfalls für die anderen Statuen, den Kasseler Apollo, Harmodios aus der Gruppe der athenischen Tyrannenmörder und zwei Skulpturen, die kein Modell erkennen lassen, aber dennoch mit den anderen Statuen harmonisieren. Dadurch bildet die photo opportunity eine besondere Form der Abstraktion, die durch eine Mischung von antiken (Statuen, klassische Architektur der Exedra, römische Ziffern) und modernen Elementen (Podest) ein Piktogramm des Sports konstruiert.

Ein weiteres Beispiel für die Strategie der Abstraktion und die Gestaltung visueller Piktogramme stellt das Restaurant Acrópolis im Herzen des Themenbereichs dar. Das Gebäude ist ein architektonisches Amalgam, das das bereits angesprochene paradigmatische Modell eines klassischen griechischen Tempels (in diesem Fall eines ionischen exastilos prostylos-Gebäudes) mit einer Reproduktion der Vorhalle der Karyatiden aus dem athenischen Erechtheion verbindet.

49 Diese Ikonografie ist von Astérix aux Jeux Olympiques (1968) popularisiert worden.

50 Vgl. WeSEnBerg, 1998, S. 61. 
Die Ansammlung der einzelnen Gebäude und dekorativen Elemente bildet insgesamt ebenfalls ein Piktogramm, nämlich das der griechischen Polis. Dabei handelt es sich weder um Athen noch um eine andere griechische Stadt, sondern um eine , synthetische“ Polis, in der Elemente aus der archaischen, klassischen und hellenistischen Zeit zusammenkommen.

Um ein möglichst hohes immersives Potenzial zu erreichen, ist der Themenbereich visuell von den anderen so weit wie möglich abgegrenzt. Aufgrund der Hügellage unvermeidbare Ausblicke werden thematisch integriert: Die erhöhte Lage der Polis wird als Akropolis konstruiert, von der man einen Ausblick auf das Mittelmeer hat, genau wie von der Akropolis Athens. Wie auch in Disneyland wird zusätzlich Musik eingesetzt, um möglichst viele Sinne anzusprechen. Selbstverständlich handelt es sich dabei nicht um eine Rekonstruktion antiker griechischer Musik, da diese nur Spezialisten bekannt sein dürfte und von einem breiteren Publikum kaum erkannt werden würde. Stattdessen wurden Stücke und Gattungen ausgewählt, die vom zeitgenössischen Besucher sofort als griechisch klassifiziert werden können, vor allem Sirtaki und insbesondere (und wie zu erwarten) Theodorakis Soundtrack zu Michael Cacoyannis’ Film Zorba il Greco (1964).

Durch diesen Verweis auf einen bekannten Film wird auch der transmediale Aspekt des Themenbereichs deutlich, der sich aber besonders durch visuelle Rahmungen wie bspw. im Falle der photo opportunity oder der geschickten Eingliederung des Mittelmeer-Panoramas in Grecia äußert. Anders als Disneyland arbeitet Terra Mítica jedoch häufig mit offenen Bildformen, was vor allem auf die Grundstruktur des Parks als Rundgang zurückzuführen ist. Vom Eingang des Themenbereichs bis zur Akropolis werden die Besucher ständig mit Perspektiven konfrontiert, die sie weiter in den Park hineinziehen. Einige der dekorativen Elemente werden darüber hinaus gleichzeitig als Kulissen und Bühnenrequisiten verwendet. Während der Show Troya, la Conquista etwa verwandelt sich so ein Teil des Themenbereichs in eine Freiluft-Theaterbühne.

Die Verwendung multi- und transmedialer Mittel (hier insbesondere in der Form der Shows) erlaubt es auch, auf den paradigmatischen und identitätsstiftenden griechischen Kulturraum bestimmte konservative Wertvorstellungen (Ehre, Rache, Maskulinität, kriegerische Tugenden) zu projizieren und dadurch zu universalisieren und zu naturalisieren. Dieser Wertekanon, der so etabliert wird, konsolidiert sich in den anderen Bereichen und erweist sich letztendlich als explizit auf die spanische Identität bezogen. Griechenland wird deshalb durch diese Formen der Geschichtsdarstellung in seiner Rolle als zentraler 
Entwicklungsschritt und elementarer Grundpfeiler der westlichen, europäischen und spanischen Identität bestätigt.

\section{Fazit}

Geschichtsdarstellung in Themenparks ist ein komplexes Phänomen, das intensiv an den kulturellen Rezeptionskontext gebunden ist. Demzufolge werden nicht nur verschiedene Epochen, Motive, Themen bevorzugt oder vernachlässigt, sondern es werden auch dieselben Elemente unterschiedlich gedeutet und rezipiert. Dies lässt sich bspw. bei Themenparkketten (wie Disneyland oder den Universal Studios) beobachten, die ein ähnliches Grundmuster an verschiedene Publikumskontexte anpassen. Main Street, U.S.A. in Disneyland, Kalifornien hat so eine ganz andere Funktion als etwa der entsprechende Themenbereich in Tokyo Disneyland, der World Bazaar. Auch wenn dieselbe Epoche oder derselbe Kulturraum in verschiedenen Parks, die nicht ökonomisch miteinander verbunden sind, dargestellt werden, kann man eine stetige Umdeutung und Anpassung an die Ziele und Prinzipien (und an die kulturellen und politischen Kontexte) der einzelnen Anlagen feststellen. Während Grecia in Terra Mítica zur Konstruktion einer spanisch dominierten ,Mittelmeeridentität" in Abgrenzung von der, europäischen Identität" beiträgt, wird der griechische Bereich im Europa-Park in Rust zur Identifizierung eines Grundpfeilers der gemeinsamen europäischen Kultur funktionalisiert.

Was synchron wie auch diachron beobachtet werden kann, sind deshalb immer geschichtstransformatorische Dynamiken, die Neukonzeptionalisierungen von konstruierten Vergangenheiten, teilweise sogar die Konstruktion von ,neuen' Vergangenheiten, und deren direkte Erlebbarkeit produzieren. Diese Produkte müssen jeweils in ihrem Entstehungskontext untersucht und verstanden werden, wobei nicht vergessen werden darf, dass ihre Existenz die nachfolgenden Formen der Rezeption und Transformation beeinflussen werden. 


\section{Literatur}

Agnew, VANESSA, History's Affective Turn. Historical Reenactment and its Work in the Present, in: Rethinking History 11, 3 (2007), S. 299-312.

AvILA, ERIC, Popular Culture in the Age of the White Flight. Fear and Fantasy in Suburban Los Angeles, Berkeley 2004.

BAUDRILlaRD, JEAN, Simulacres et simulation, Paris 1981.

Bergemann, Lutz, u. a., Transformation. Ein Konzept zur Erforschung kulturellen Wandels, in: Transformation. Ein Konzept zur Erforschung kulturellen Wandels, hg. von HARTMUT BÖHME u. a., München 2011, S. 39-56.

BIEGER, LAURA, Ästhetik der Immersion. Raum-Erleben zwischen Welt und Bild. Las Vegas, Washington und die White City, Bielefeld 2007.

BRYMAN, ALAN, Disney and his Worlds, London 1995.

CARLÀ, FILIPPO, Historische Quellen, literarische Erzählungen, phantasievolle Konstruktionen. Die vielen Leben der Theodora von Byzanz, in: Transkulturelle Dynamiken. Aktanten - Prozesse - Theorien, hg. von JuTTA ERNST/ FLORIAN FREITAG, Bielefeld 2014, S. 31-62.

ClüVER, Claus, Intermediality and Interarts Studies, in: Changing Borders. Contemporary Positions in Intermediality, hg. von JENS ARVIDSON u. a., Lund 2007, S. 19-37.

De Groot, Jerome, Consuming History. Historians and Heritage in Contemporary Popular Culture, London 2009.

Finch, Christopher, The Art of Walt Disney. From Mickey Mouse to the Magic Kingdoms and Beyond, New York 2011.

FJellman, Stephen M., Vinyl Leaves. Walt Disney World and America, Boulder 1992.

FoucAult, Michel, Des espaces autres, in: Foucault - Dits et écrits 19541988 (Collection Quarto), 2 Bde., Bd. 2, 1976-1988, hg. von DANIEL DEFERT, Paris 2005, S. 1571-1581.

HÖLSCHER, LUCIAN, Die Zukunft - ein auslaufendes Modell neuzeitlichen Geschichtsbewußtseins?, in: Die Zeit im Wandel der Zeit, hg. von HANSJOACHIM BIEBER u. a., Kassel 2002, S. 129-146.

Holtorf, Cornelius, From Stonehenge to Las Vegas. Archeology as Popular Culture, Lanham 2005.

DERS., The Presence of Pastness. Themed Environments and Beyond, in: Staging the Past. Themed Environments in Transcultural Perspectives, hg. von JUDITH SCHLEHE u. a., Bielefeld 2010, S. 23-40. 
JordanOVA, Ludmilla J., History in Practice, 2. Aufl., London 2006.

KAGELMAnN, H. JÜRGEN, Themenparks, in: Tourismuspsychologie und Tourismussoziologie. Ein Handbuch zur Tourismuswissenschaft, hg. von HEINZ HAHN/DEMS., München 1993, S. 407-416.

KIng, MARgaret J., Disneyland and Walt Disney World. Traditional Values in Futuristic Form, in: Journal of Popular Culture 15, 1 (1981), S. 116-140.

LAINSBURY, ANDREW, Once upon an American Dream. The Story of Euro Disneyland, Lawrence 2000.

LeE, Newton/MadeJ, Krystina, Disney Stories. Getting to Digital, New York 2012.

LUKAS, ScOTT A., A Politics of Reverence and Irreverence. Social Discourse on Theming Controversies, in: The Themed Space. Locating Culture, Nation, and Self, hg. von DEMS., Lanham 2007, S. 271-293.

DERS., Theme Park, London 2008.

Marling, Karal AnN, Disneyland, 1995. Just Take the Santa Ana Freeway to the American Dream, in: American Art 5, 1-2 (1991), S. 168-207.

PHILIPS, DEBORAH, Narrativised Spaces. The Functions of Story in the Theme Park, in: Leisure/Tourism Geographies. Practices and Geographical Knowledge, hg. von DAVID CROUCH, London 1999, S. 91-108.

SAMUEL, RAPHAEL, Theatres of Memory. Past and Present in Contemporary Culture, 2. Aufl., London 2012.

SchleHE, Judith u. a. (Hg.), Staging the Past. Themed Environments in Transcultural Perspectives, Bielefeld 2010.

ShafFer, JoshuA C., Discovering the Magic Kingdom. An Unofficial Disneyland Vacation Guide, Bloomington 2010.

SHANnON, LEONARD, Disneyland: Dreams, Traditions and Transitions, Burbank o. J.

SHRAGER, MONIQUE, Euro Disneyland, Burbank 1994.

STEINECKE, ALBRECHT, Erlebniswelten und Inszenierungen im Tourismus, in: Geographische Rundschau 2 (2000), S. 42-45.

Wallace, Mike, Mickey Mouse History. Portraying the Past at Disney World, in: Radical History Review 32 (1985), S. 33-57.

WESENBERG, BURKHARDT, Für eine situative Deutung des polykletischen

Doryphoros, in: Jahrbuch des Deutschen Archäologischen Instituts 112 (1998), S. 59-75.

WIENER, JON, Tall Tales and True, in: The Nation, 31.1.1994, S. 133-135. 